\title{
6
}

\section{Biological concept of self-organization for dynamic shop-floor configuration}

\author{
Jari Vaario ${ }^{\dagger}$ and Kanji Ueda ${ }^{\ddagger}$ \\ ${ }^{\dagger} N T T$ Human Interface Laboratories \\ Hikarinooka 1-1, Yokosuka, 239 JAPAN \\ Tel: +81-468-59-8119, Fax: +81-468-59-2332, \\ E-mail: jari@nttcvg.hil.ntt.co.jp \\ $\ddagger$ Department of Mechanical Engineering, Kobe University \\ Rokko Nada, Kobe 657, JAPAN \\ Tel: +81-78-803-1123, Fax: +81-78-803-1131, \\ E-mail: ueda@mech.kobe-u.ac.jp
}

\begin{abstract}
In this paper we propose a new simulation method for dynamic shop-floor layout configuration inspired by biological systems. Simulating the mechanisms of biological systems we explore the possibilities for self-organizing an assemble line, where the shop-floor layout is the result of self-organization. This research is a part of the wider concept of biological manufacturing systems. The results show that the proposed ideas are feasible for modeling a small scale assembly tasks.
\end{abstract}

\section{Keywords}

Intelligent manufacturing system, biological manufacturing system, self-organization, dynamic configuration, shop-floor layout

\section{INTRODUCTION}

A new research field in computer science, artificial life (Langton, 1989; Brooks and Maes, 1994; Morán, Moreno, Merelo and Chacón, 1995), has matured to be applied to various engineering problems. In this paper some results of artificial life research will be applied to manufacturing domain following the general proposal of Biological Manufacturing Systems (Ueda, 1994; Ueda and Ohkura, 1995; Vaario and Ueda, 1996b; Ueda, Vaario and Ohkura, 1997).

Artificial life field has a long history of theoretical thinking. However, it was 
until late 1980s when the current computer technology provided the needed possibilities for modeling artificial realities and studying life-like behaviors.

The focus on the artificial life research has been on how to create autonomous behaviors based on local interactions (bottom-up approach) rather than defining the global behavior and decomposing it to lower level actions (top-down approach). The bottom-up approach leads obviously to a local optimization. However, the object of this research is not to provide a global optimization, but to provide a method for dynamic and continuous adaptation. As it is often the case in advanced manufacturing systems, the key concept is not any more the global optimization, but the capability of responding immediately to various disturbances - internal or external.

The approach defining only the bottom level interactions and letting the global behavior to emerge could be done in various ways. One important aspect in this is how the environment participates into this process. Having a mechanism that is effected at least partly by the environmental factors, we have a method that could be applied to various engineering problems of modeling adaptation.

The goal is not to design directly the final product, but to design a process that will produce it. When the environment is part of this process, the environmental changes will effect directly to the final product, i.e. the system adapts to the environmental changes. The result is a kind self-adaptable system capable of maintaining a stable, but flexible to new disturbances, organization. The biological systems are a good example of this kind of systems.

When we apply this concept to manufacturing systems, the problem becomes to what is this construction process, and how our intentions could be defined within this process. In this paper the we describe one possible implementation, and its computer simulation, for the construction process. The intention part is implemented by combining the top-down (design of products, i.e. requirements) and bottom-up (the actual manufacturing processes, i.e. capabilities) approaches.

\section{GENERAL CONCEPT}

In this paper the biological concept is applied at the metaphoric level to the manufacturing systems. The biological life cycle is shown on the left side of Figure 1. This cycle could be divided into two parts based on what level operations are considered. The evolutionary part describes how the genetic information evolves by the natural selection and genetic modifications. The developmental part describes the individual sytem to take a shape and maintaining this in case of disturbances.

This cycle could be applied to manufacturing concept at various levels (Vaario and Ueda, 1996a). In this paper we concentrate on applying this on a production system, where the cycle could be viewed as shown in Figure 1 right.

The intersection of these cycles is at the shop-floor with a problem to de- 

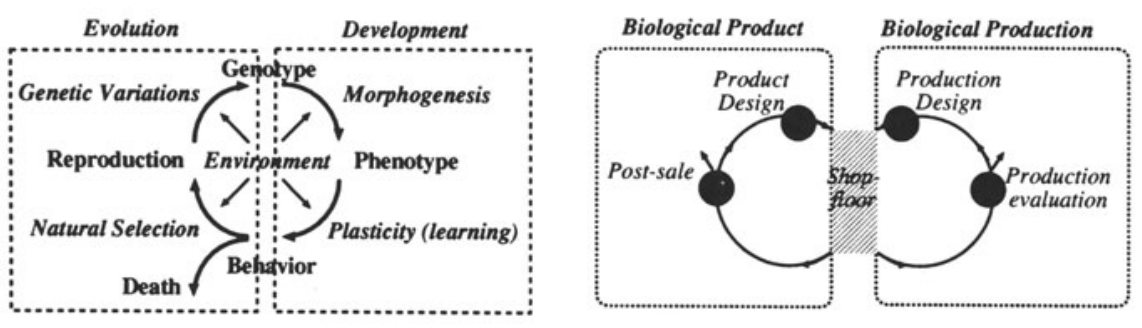

Figure 1 Biological life cycle and its metaphoric presentation of manufacturing systems.

termine where the products are processed. In other words, the problem is where the product should go and where the manufacturing units should locate to provide an efficient manufacturing environment, especially when one consideres the conditions to be dynamic. So how to design a system capable of creating this from random locations of manufacturing units, maintaining this in the case of distrubances such as malfunctions in manufacturing units, and reorganizing this in cases where the product will change.

\subsection{Self-Organization}

The main focus is on to show how the shop-floor operations could adapt using self-organization principles with parameters adjusted through these cycles. The following inclusive list indicates the major points how self-organization could contribute to adaptive manufacturing systems. Here the manufacturing systems are viewed mainly as cellular systems consisting of transporters (carrying products) and manufacturing units (processing the products).

- Self-organization of shop-floor layout: Both transporters and manufacturing units are movable. Gradually a layout is emerging from the interactions between transporters and manufacturing units by minimizing the transportation distances.

- Dynamic schedule: The manufacturing units are static and the goal is to find out a schedule of transporting products between manufacturing units. The dynamic schedule means that the schedule is capable of changing in the case of malfunction of a manufacturing unit, or transporter.

- Adaptation to product demand: The manufacturing system will adapt to the changes in product demand by re-organizing production line(s), increasing the number of manufacturing units and transporters, as well as modifying their capabilities.

- Adaptation to production changes: The manufacturing units are described by processing capabilities, and products by processing requirements. When a requirement is changed the production should change accordingly. 
In this paper the self-organization of shop-floor layout is described with an example of dynamic configuration of a bicycle assmbly line.

\subsection{Evaluation methods}

The evaluation of the properties of adaptive manufacturing systems is a difficult topic. It is not clear how to measure adaptation and flexibility. The evaluation methods are still to be developed, but the following approaches are considered this far.

- The cumulative distance of parts traveled before the final product was finished.

- The cumulative waiting time of parts before processed.

- The time consumed to finish the final product. This is a combination of traveling, waiting, and assembling times.

- The idle time of each production unit.

- The lapsed time from entering a new product description and receiving a new product.

- The capability to change the production when the consumption changes.

- The capability of reconfiguration in the case of malfunctions at some production units.

\section{SIMULATION METHOD}

Simulation method is based on a gradient field modeling method. For a complete description see (Vaario, 1994; Vaario and Shimohara, 1995). The idea is to model a virtual space where the forces (gradient field models) effect the manufacturing cells and transporters guiding them in the space and determining their relative locations at each moment, i.e. to create a configuration of an assemble line.

The forces are the only means to determine the configuration, and they are also the only method for maintaining the configuration. The forces act locally and thus implement a bottom-up modeling method.

Each manufacturing unit has attraction fields according to its capabilities. On the other hand, each part is sensitive for some attraction field that would satisfy its needs. Thus the capabilities of manufacturing units and the needs to parts will be matched by simulating continuously the effect of force fields. This is illustrated in Figure 2.

The continuous simulation approach provides two major advantages over traditional systems: 1) the production line configuration is flexible in the means that the simulation creates and maintain the configuration based on the local information of "needs to be processed" and "capabilities to process"; 


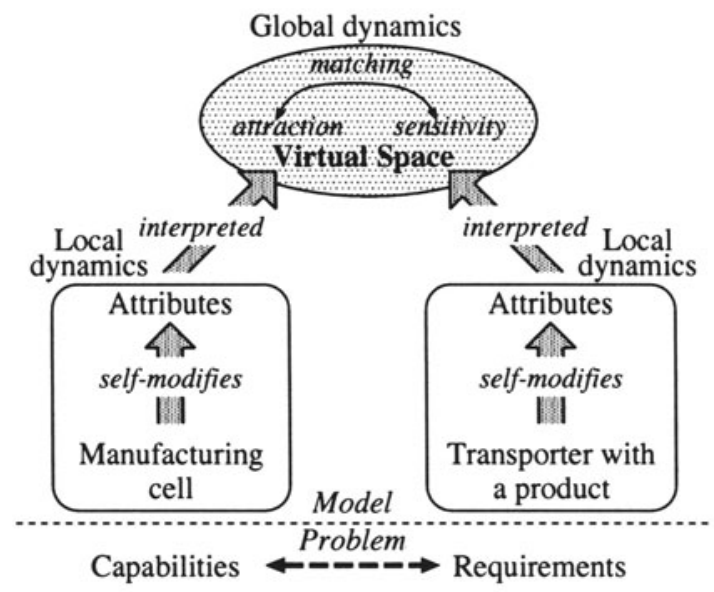

Figure 2 The local dynamics vs. global dynamics.

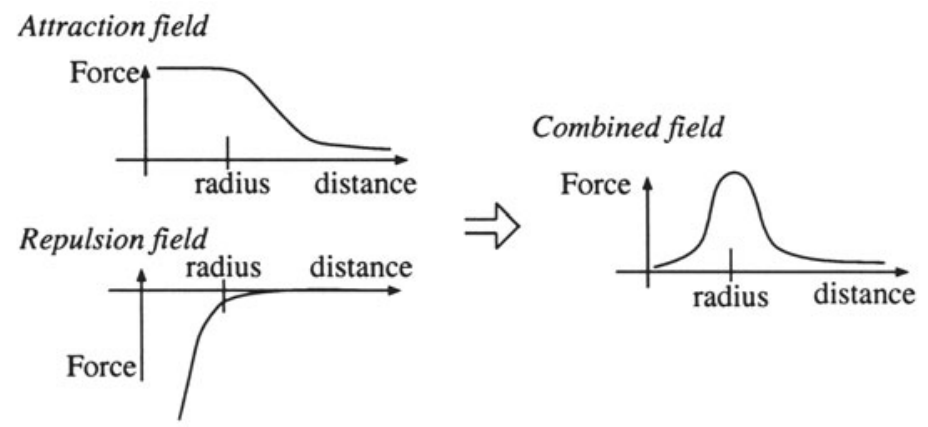

Figure 3 Attraction and repulsion fields with the combined force field.

2) compared with a static configuration of such a line, the re-configuration (adaptation) is continuous and autonomous without an explicit decision of re-configuration.

A simple form of the used attraction and repulsion force fields as a function of the distance are shown in Figure 3. These fields describe the scalar value of the force vectors effecting between parts and production units. The forces effect mutually, i.e. if a production unit draws a part for it, a similar force also effects from the part to the production unit. The actual movement is relative to the mass of each unit.

Although not applied yet in the field of manufacturing cells, the simulation method allows modeling of dynamic force fields. Thus in the case where a production unit can not receive its input, it can increase its attraction fields. In the opposite case, the field value could be reduced if there is plenty input available. This enable a competition for input between production units. 

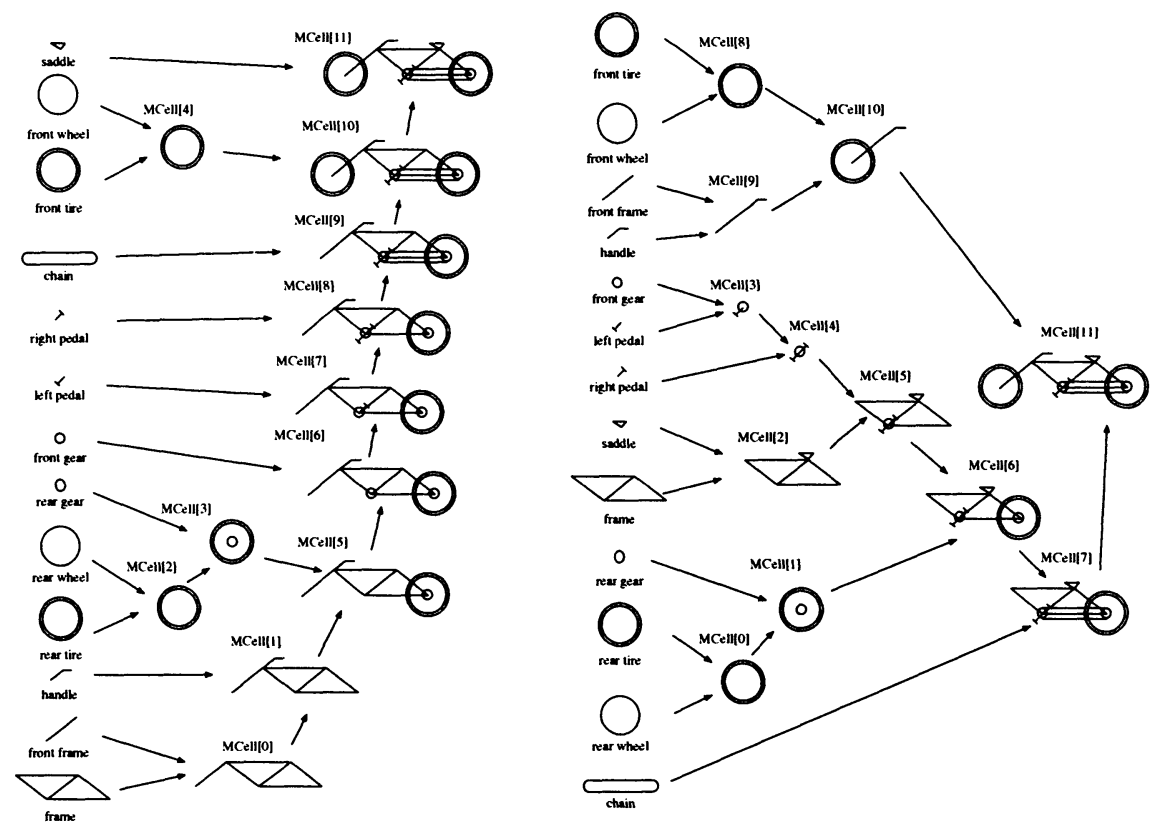

Figure 4 Product definitions for a 'flat' and 'hierarchical' production.

\section{A TEST CASE: BICYCLE ASSEMBLE}

In the following a detailed description of using the above model for a test case of a bicycle assemble line is given.

\subsection{Product Description}

The product model of a bicycle consists of following thirteen kinds of parts: frame, front frame, handle, front wheel, front tire, rear wheel, rear tire, front gear, rear gear, right pedal, left pedal, chain and saddle. In addition there are several combinations of these parts according to what order the parts are put together.

Two bicycle models as shown in Figure 4 are tested. The first one (on left) is designed to be 'flat', and the second one is designed to be 'hierarchical'. The difference should be visible in the resulting shop-floor layout.

\subsection{Assemble cell}

Each assemble cell (see Figure 5 right) generates attractions for two input corresponding to the above product description. When both input are received 


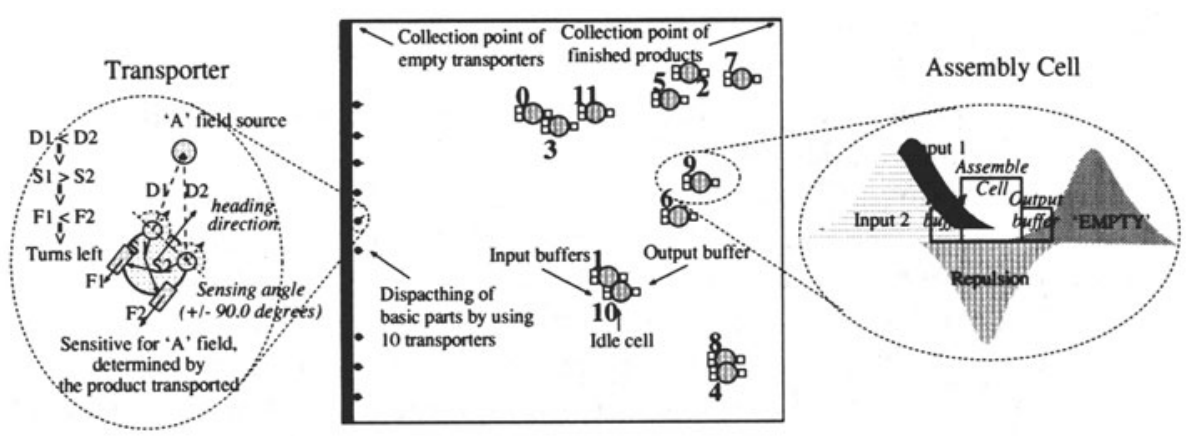

Figure 5 Initial configuration where assembly cells are at random locations (middle) with detail illustrations of assembly cells (left) and transporters (right).

they are assembled (taking a constant time) and the output is put into output buffer. The output will be an input to an other assemble cell according to the product description, or could be an input to the collection point of finished bicycles. A repulsion field is used to prevent assemble cells to overlap each others.

The field dynamics involves only on-off timing. When input buffers are full the input attraction is turned off. When the output buffer is full, then an 'empty transporter' attraction is raised in order to get an empty transporter to pick the output. This will be turned off once the output buffer will become empty.

\subsection{Transporter}

Each part of a bicycle moves on a transporter (see Figure 5 left) in the shop floor. Transporters do not have particular trails, but they can move freely on the shop floor space. They became sensitive to the attraction fields according to the parts they carry. If transporters do not carry any products they are sensitive to 'empty transporter' attractions. This attraction is generated by the factory dispatcher, that dispatches parts to the factory at the left side wall, or by the output buffers of assemble cells. If there are no attractions detected the transporters are going around indicating their idle state.

\section{SIMULATION RESULTS}

The following example provides the first results of the simulation method applied to self-organize the shop-floor layout (Fujii, Vaario and Ueda, 1997). The method has been used also to emerge a dynamic schedule for a task of drilling printed boards (Vaario and Ueda, 1997). 


\subsection{Initial values}

The initial shop-floor space with random locations of assembly cells is illustrated in Figure 5 middle.

The dispatching of basic parts was set at the left wall in some distance from each others. The wall had a repulsion field to prevent assemble cells to go too far on it. The basic parts were allocated for empty transporters in linear order so that there was all time the correct ratio of basic parts on the shop floor. The collection of empty transporters to pick up next parts was set on the left-top corner. The collection point of finished products was set at the right-top corner.

The initial configuration of assemble cell was set random at the right half of the shop floor area. This was to give the assemble cells time to direct their movement toward the correct locations before being crowded at the left side wall. Each assemble cell was given the type of its input to attract. The assemble time was set same for each task.

The number of available transporters was set to ten. Transporters did not use any repulsion field, thus they were able to cross assemble cells and other transporters. Various parameters to control the movements were found by trial-and-error approach. The evaluation criteria for this was simply to produce 'nice-looking' behavior.

\subsection{Self-organization of production lines}

As a simulation result, the self-organization process of assemble cells on the shop-floor is shown in Figure 6 (left) for the first type of production. The initial configuration is random at the right half of shop-floor space. As the simulation progresses, the shop floor layout is changing gradually. The most changes are caused in very early simulation steps. A near-to-stabile configuration is reached already when only a few bicycles are fully assembled. After about fifteen bicycles are assembled the configuration starts to oscillate and no relative positions are changed anymore.

In the second case the floor layout is reached in same manner from same initial configuration. However, this time the configuration is completely different (Figure 6 (right)) corresponding a different assemble order. The resulting configuration demonstrates the capability of self-organizing different production configurations with relative small changes in the product description. This provides furthermore the possibility to test what kind of production order is the most efficient.

As one can observe the both shop floor layouts correspond to the product descriptions given in Figure 4 as expected. In order to to achieve a good layout the dispatching positions of basic parts have been ordered differently 

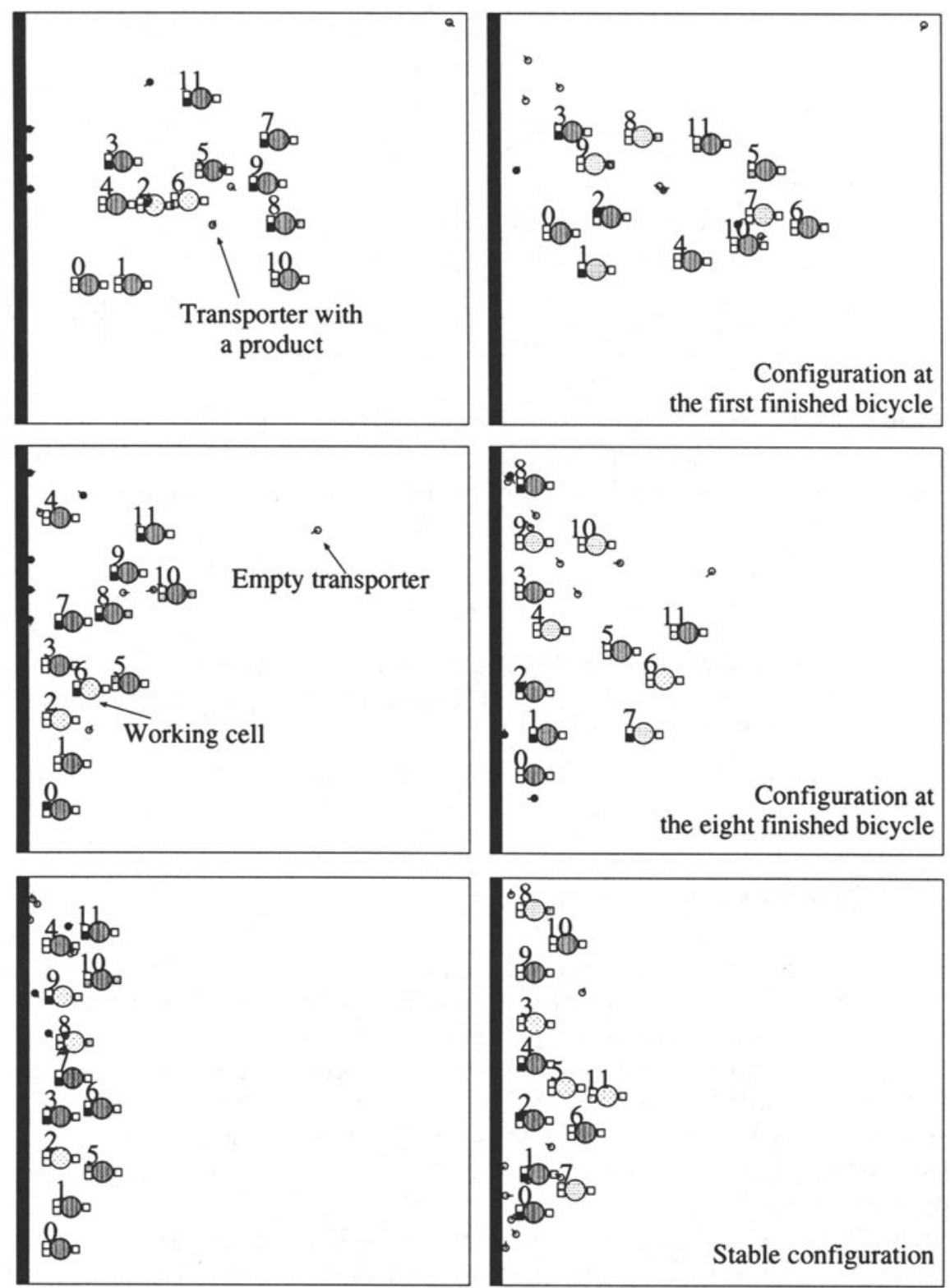

Figure 6 Self-configuration process of the 'flat' (left) and the 'hierarchical' (right) production line. The first snapshot is taken when the first product is finished, the second when the eighth product is finished, and the third snapshot is taken when a stabile configuration is reached. 

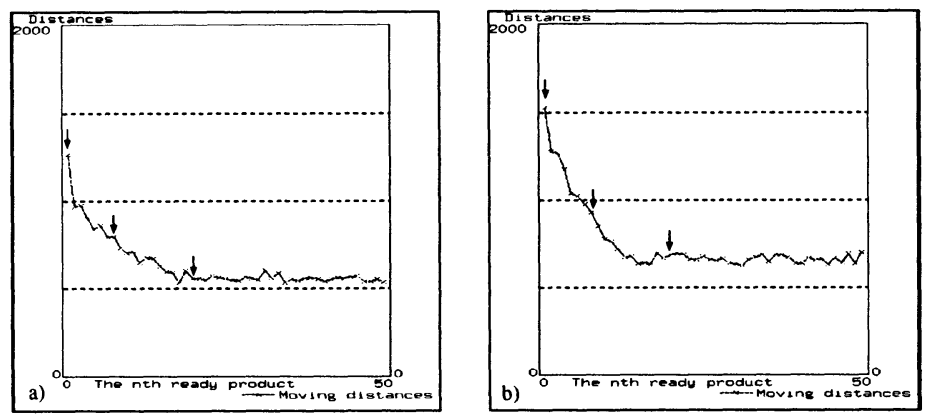

Figure 7 The transportation distance between assemble cells for 'n'th products by the a) 'flat', and b) 'hierarchical' production line. Arrows in the graphs show the corresponding snapshots of configuration in Figure 6 .

for both cases. Also the numbering of cells is slightly different because they are performing different assemble tasks (cf. Figure 4 for corresponding numbers).

\subsection{Evaluation and discussion}

The graph for evaluating the result, a process of self-configuration during the simulation of 50 first bicycles by the 'flat' production line and by the 'hierarchical' production line, is shown in Figure 7.

The graphs represent the cumulated transportation distances for the subparts of 'n'th bicycle. The first and last transportation distances are not included in order to measure the relative configuration of manufacturing cells. This distance is supposed to decrease when a better configuration is reached, as shown in the graphs.

The fluctuation in the stabile configuration is shown in the graphs by variations in the measured distances. Although this fluctuation is unnatural, it could be understand as a capability of re-configuration in the case of changing conditions.

The same transportation distance is achieved in almost every time at latest by the twelfth product assembled. In some rear cases the manufacturing cells are blocking temporally movement of each others causing a step in the curve.

Although we cannot guarantee an optimal configuration, the achieved configuration is near the optimal. The main merit of the method is, however, the fact that the configuration is reached automatically with a capability for re-configuration in the case of environmental perturbations. 


\section{CONCLUSION}

This paper described a novel method for self-organization of an assemble line. The main focus was on the concepts, and the actual results of simulations and their analysis were left for the future work. The main message on this paper is that we should imitate the nature's production system, and especially its capability of self-organizing, self-adapting, self-repairing, self-maintaining, etc.

The other projects have considered using the approach on dynamic scheduling problems (Vaario and Ueda, 1997; Ueda et al., 1997; Ueda and Vaario, 1997). Also an interactive factory animation has been tried with a virtual reality implementation (Vaario, Fujii, Scheffter, Mezger and Ueda, 1997). The selected approach of applying various biological concepts of self-organization is promising, but a lot of work remains in developing the analysis and measurement methods for the dynamics simulations.

\section{ACKNOWLEDGMENTS}

The authors thank Mr. Fujii for his help on implementing the simulations of the example.

\section{REFERENCES}

Brooks, R. and Maes, P., eds (1994), Artificial Life IV, The MIT Press.

Fujii, N., Vaario, J. and Ueda, K. (1997), Potential field based simulation of self-organization in biological manufacturing systems, in 'Proceedings of Manufacturing System Design'97, May 14-16, 1997, Magdeburg, Germany', LOGIS, Stuttgart.

Langton, C. G., ed. (1989), Artificial Life, Addison-Wesley Publishing Company.

Morán, F., Moreno, A., Merelo, J. J. and Chacón, P., eds (1995), Advances in Artificial Life, Springer.

Ueda, K. (1994), Biological-oriented paradigm for artifactual systems, in '1994 Japan-U.S.A. Symposium on Flexible Automation', ISCIE/ASME, pp. 1263-1266.

Ueda, K. and Ohkura, K. (1995), A biological approach to complexity in manufacturing systems, in 'Proc. of the 27th CIRP International Seminar on Manufacturing Systems', Ann Arbor, pp. 69-78.

Ueda, K. and Vaario, J. (1997), The biological manufacturing system: Adaptation to growing complexity and dynamics in manufacturing environment, 29th CIRP International Seminar on Manufacturing Sytems, May, 1997, Osaka, Japan, pp. 39-44.

Ueda, K., Vaario, J. and Ohkura, K. (1997), Modeling of biological manufac- 
turing systems for dynamic reconfiguration, Vol. 46/1, Annals of the CIRP, pp. 343-346.

Vaario, J. (1994), Modeling adaptative self-organization, in Brooks and Maes (1994), pp. 313-318.

Vaario, J., Fujii, N., Scheffter, D., Mezger, M. and Ueda, K. (1997), Factory animation by self-organization principles, in N. M. Thalman, ed., 'International Conference on Virtual Systems and MultiMedia 1997 (VSMM'97)', Springer-Verlag. (to appear).

Vaario, J. and Shimohara, K. (1995), On formation of structures, in Morán et al. (1995), pp. 421-435.

Vaario, J. and Ueda, K. (1996a), Biological concept of self-organization in flexible automation systems, in N. Okino, H. Tamura and S. Fuji, eds, 'Proceedings of APMS'96 (Advanced Product Management Systems)', November 4-6, 1996, Kyoto, Japan, pp. 33-38.

Vaario, J. and Ueda, K. (1996b), Self-organization in manufacturing systems, in '1996 Japan-USA Symposium on Flexible Automation', July 7-10, 1996, Boston, MA, pp. 1481-1484.

Vaario, J. and Ueda, K. (1997), An emergent method for dynamic scheduling, The Second World Congress on Intelligent Manufacturing Processes \& Systems, June 10-13, 1997, Budapest, Hungary, pp. 187-198.

\section{BIOGRAPHY}

Jari Vaario received M.S. degree in engineering from Helsinki University of technology, Finland, in 1985. He was awarded Doctor in Engineering degree by the University of Tokyo, Japan, in 1994 while working at ATR Human Information Processing Laboratories, Kyoto, Japan. From 1995 to 1997 he was a full professor at Nara Women's University, Japan, and from 1997 he has been an invited professor at NTT Human Interface Laboratories, Yokosuka, Japan. His research interests include modeling and simulating self-organization and emergent phenomena, and applying these into various areas.

Kanji Ueda graduated from Master Course of Engineering, Osaka University, Japan in 1972. He joined Department of Mechanical Engineering, Kobe University, as a Research Associate, after his graduation. He obtained the degree of Doctor of Engineering in 1978 from Osaka University. He joined Kanazawa University as an Associate Professor in 1980, and later he became a Full Professor at the same university in 1988. He rejoined Kobe University as a Full Professor in 1990, and he is now Head of the Unit of Intelligent Machines and Manufacturing System at Department of Mechanical Engineering and concurrently the Director of Kobe University Information Processing Center. 